\title{
Is additional pulsatile pulmonary blood flow beneficial to patients with bidirectional Glenn?
}

\author{
Sunita J. Ferns, MD, MRCPCH, ${ }^{\mathrm{a}}$ Chawki El Zein, MD, ${ }^{\mathrm{b}}$ Kanwar Multani, MBBS, ${ }^{\mathrm{a}}$ Imran Sajan, MD, ${ }^{\mathrm{a}}$ \\ Sujata Subramanian, MD, ${ }^{\mathrm{b}}$ Anastasios C. Polimenakos, MD, ${ }^{\mathrm{c}}$ and Michel N. Ilbawi, $\mathrm{MD}^{\mathrm{b}}$
}

Objective: To compare the results of bidirectional Glenn when performed with or without pulsatile pulmonary blood flow in a cohort of patients with a single ventricle.

\begin{abstract}
Methods: Records of 212 patients undergoing staged single ventricle palliation during a 10-year period were retrospectively reviewed. Of those, 103 (33 in pulsatile group A and 70 in nonpulsatile group B) were selected.

Results: Demographics and pre- and intraoperative variables were comparable for both groups. There was no difference in oxygen saturations immediately after the bidirectional Glenn in the 2 groups. The duration and output of chest tube drainage, incidence of chylothorax, and total length of stay was higher in group A. There was no difference in the number of diuretics or oxygen requirement upon discharge between groups. Pre-Glenn measurements showed a mean McGoon ratio in group A of 1.5 (1.46-1.57) and in group B of 1.59 (1.53-1.7) $(P=.11)$; however, there was a significant difference in the ratio between groups at pre-Fontan measurements: group A, $1.76(1.73-1.79)$ and group B, $1.6(1.53-1.66)(P<.05)$. At pre-Fontan measurements there was a significant difference in mean pulmonary artery pressure between group A (14 mm [12.8-15.2]) and group B $(10 \mathrm{~mm}[9.7-11])(P<.05)$ and a trend toward higher incidence of venovenous collaterals in group $\mathrm{A}$. There was no perioperative or interstage mortality in either group.
\end{abstract}

Conclusions: Pulsatile bidirectional Glenn is associated with better pulmonary artery growth, which might improve long-term outcomes after Fontan. However, it was associated with a higher postoperative complication rate. (J Thorac Cardiovasc Surg 2013;145:451-4)

Controversy continues regarding whether additional sources of pulmonary blood flow are beneficial in combination with the bidirectional Glenn procedure (BDG). The benefits of additional anterograde pulsatile flow include potential growth of pulmonary arteries, ${ }^{1-5}$ improvement in arterial oxygen saturation, ${ }^{6-8}$ and prevention of development of pulmonary arteriovenous malformations. ${ }^{8}$ However, previous work has documented an increase in systemic venous pressure and associated complications with additional pulsatile blood flow after BDG. ${ }^{9,10}$ The objective of our study was to delineate the beneficial effects of additional pulmonary blood flow following the pulsatile BDG.

\footnotetext{
From the Divisions of Pediatric Cardiology and Pediatric Cardiothoracic Surgery, ${ }^{\mathrm{b}}$ Hope Children's Hospital, Oak Lawn, Ill; and Division of Pediatric Cardiothoracic Surgery, ${ }^{\mathrm{c}}$ Rush University Hospital, Chicago, Ill.

Disclosures: Authors have nothing to disclose with regard to commercial support.

Read at the 38th Annual Meeting of The Western Thoracic Surgical Association, Maui, Hawaii, June 27-30, 2012.

Dr Ferns' current affiliation is the Department of Medicine, Penn State Medical Center. Hershey, Pa. Dr Polimenakos' current affiliation is The Heart Center for Children at J Weis Children's Hospital-Geisinger Clinic, Danville, Pa.

Received for publication June 20, 2012; revisions received Oct 8, 2012; accepted for publication Nov 6, 2012.

Address for reprints: Sunita J. Ferns, MD, MRCPCH, 412 Hallmark S, Hershey, PA 17033 (E-mail: sjulianaferns@yahoo.com). 0022-5223/\$0.00

Published by Elsevier Inc. on behalf of The American Association for Thoracic Surgery

http://dx.doi.org/10.1016/j.jtcvs.2012.11.027
}

\section{MATERIALS AND METHODS Patient Selection}

Records of 212 patients undergoing staged single ventricle palliation during a 10-year period between January 1, 2000, and December 31, 2010, were retrospectively reviewed. For each pulsatile Glenn (group $\mathrm{A}, \mathrm{n}=33$ ) 2 consecutive patients undergoing a nonpulsatile Glenn were selected as control subjects (group $\mathrm{B}, \mathrm{n}=70$ ), thereby eliminating the bias inherent in clustering or surgeon's preference.

\section{Clinical Data}

Demographic data, a detailed anatomic diagnosis, and previous surgical or interventional procedures were noted. Perioperative data included details of surgery performed, crossclamp time, and bypass time. Postoperative characteristics included duration of ventilation, saturations in the immediate postoperative period, duration and amount of chest tube drainage, complications, length of intensive care unit stay, and duration of hospitalization. Characteristics at the time of discharge, such as need for home oxygen and medications at discharge and readmissions during the second interstage period, were reviewed. All patients underwent a predischarge echocardiogram and a follow-up echocardiogram evaluation every 3 to 6 months after discharge.

\section{Operative Technique}

BDG was performed through a median sternotomy incision. The superior vena cava to right pulmonary artery anastomosis was performed on a beating heart with cardiopulmonary bypass, using interrupted prolene sutures. After weaning the heart from cardiopulmonary bypass, the antegrade pulmonary blood flow was adjusted by applying a pulmonary artery band in patients with native pulmonary stenosis, or by tightening a previously applied pulmonary artery band. The band was adjusted until the mean Glenn pressure was around 12 torr. We use a mean pressure of 12 torr for the Glenn because a pressure of 9 to 14 torr has been associated with excellent outcomes in 


\section{Abbreviation and Acronymn \\ $\mathrm{BDG}=$ bidirectional Glenn}

\begin{abstract}
our experience. Any other source of pulmonary blood flow, like patent ductus arteriosus and/or systemic to pulmonary shunt, was taken down. In 6 patients with a Sano, antegrade flow was maintained by banding the right ventricle to the pulmonary artery shunt. The decision to maintain pulsatile flow was based on preoperative measurements of the pulmonary arteries and subjective assessment of the decreased capacity of the pulmonary vascular bed. Echocardiographic data were collected in the immediate postoperative period, at the time of hospital discharge from the BDG, and at the time of the pre-Fontan admission. Cardiac catheterization data were collected at the pre-BDG and pre-Fontan period, including systemic and pulmonary artery saturations, pulmonary artery pressures, pulmonary artery size, and presence of collaterals. Anteroposterior angiographic views were used to measure pulmonary artery diameter proximal to the upper lobe branch. Aortic diameter was measured at the level of the diaphragm. The McGoon ratio was calculated to correct for body surface area differences and to overcome limitations with angiographic measurements and calibrations. All measurements on the McGoon ratio for the purpose of our study were carried out by a single observer to eliminate interobserver bias. Pulmonary to systemic blood flow ratios were not reported because of the inherent error in the measurement of ratios when there is dual pulmonary blood flow.
\end{abstract}

\section{Statistical Analysis}

Data was analyzed with SPSS version 15.0 (IBM Corp, Armonk, NY). Data are expressed as mean with $95 \%$ confidence intervals in parentheses. Comparative univariate analysis was performed between the 2 groups of patients with the $t$ test for normally distributed continuous data. The $\chi^{2}$ test was used for ordinal data.

\section{RESULTS}

Demographics are shown in Table 1. There was no significant difference between group A and B in terms of age, body weight, ventricular function and systemic valve regurgitation, pulmonary artery pressures, and McGoon ratio before BDG. Anatomic diagnosis is depicted in Figure 1 with a predominant number of patients in group B having hypoplastic left heart syndrome or a variant. Most patients were extubated the day of surgery either in the operating room or within the first few hours of intensive care unit stay. Although there was a trend toward higher oxygen saturations in group A, this difference was not significant (Table 1). The duration and output of chest tube drainage, incidence of chylothorax, and total length of stay was higher in group A. There was no significant difference in the number of diuretic agents or oxygen requirement upon discharge between groups. Postoperative echocardiographic data is detailed in Table 2. There was no significant difference in the degree of ventricle or atrioventricular valve dysfunction between the 2 groups. Pre-Fontan cardiac catheterization data is detailed in Table 3. There was no perioperative or interstage mortality in either group.

\section{DISCUSSION}

The concept of 2 systemic venous in parallel connections as a source pulmonary blood flow was first introduced by
Billingsley in $1989 .{ }^{11}$ During systole, inferior vena cava blood return reaches the pulmonary arteries by pulmonary ventricular contraction and in diastole, superior vena cava flow is delivered to the pulmonary arteries by venous pressure and laminar flow. ${ }^{11}$ Optimal Fontan hemodynamics depend in part on the resistance and the capacity of the pulmonary vascular bed. Review of the literature thus far demonstrates that pulmonary artery size positively correlates with outcome after Fontan procedure. As early as 1989, Fontan and colleagues ${ }^{12}$ published their results of a multicenter study of 334 patients and concluded that dimensions of the pulmonary arteries were one of the most powerful risk factors for death with or takedown of the Fontan circulation. An increased risk is especially evident at a McGoon ratio below 1.8 and increased sharply with smaller pulmonary arteries still further. In contrast, good pulmonary artery size has been reported to improve postoperative outcome after the primary Fontan procedure. $^{12}$

Pulsatile blood flow has been shown to be an important determinant of growth of the pulmonary vascular bed and has been observed in several clinical situations such as growth of an isolated pulmonary artery after it is shunted. At a tissue level, pulsatile pulmonary blood flow plays an important role in regulating shear-stress-mediated release of endothelium-derived nitric oxide and endothelin-1. It is also important in reducing pulmonary vascular resistance by passive capillary recruitment. ${ }^{13}$ An additional advantage of pulsatile blood flow, especially when the source is forward flow from the single ventricle receiving the inferior vena cava blood flow, is the provision of hepatic factor to the pulmonary vascular bed, thus minimizing the probability of development of pulmonary arteriovenous fistulae. This is especially important if the Fontan procedure is delayed to age 4 years, as is the practice in most centers. The third advantage is provision of increased saturations during the critical period of brain growth in an infant. With improved pulmonary artery growth, there is additionally potential for an early Fontan repair, ${ }^{14}$ leading to improved systemic saturations earlier during the crucial developmental years.

Data on whether additional anterograde pulsatile flow at the time of the Fontan actually improves pulmonary artery size is limited. Whereas McElhinney and colleagues ${ }^{9}$ questioned the adequacy of pulmonary artery growth, several other groups have demonstrated an absence of regression or an actual improvement in pulmonary artery indices with the additional effects of anterograde flow. ${ }^{1-5}$ To the best of our knowledge there have been no case-control studies comparing the clinical course of pulsatile BDG with matched nonpulsatile controls. Data from our study revealed that additional anterograde pulsatile flow does have a positive significant influence on pulmonary artery size before the Fontan procedure. 
TABLE 1. Demographic and perioperative data for groups A and B

\begin{tabular}{lccc}
\hline \multicolumn{1}{c}{ Parameter } & $\begin{array}{c}\text { Group A } \\
(\mathbf{n}=\mathbf{3 3})\end{array}$ & $\begin{array}{c}\text { Group B } \\
(\mathbf{n = 7 0 )}\end{array}$ & $\boldsymbol{P}$ value \\
\hline Age at Glenn (d) & $182(155-209)$ & $176(153-200)$ & .76 \\
Male:Female & $20: 13$ & $48: 22$ & .43 \\
Weight (kg) & $5.6(5.2-6.1)$ & $5.8(5.5-6.1)$ & .2 \\
Oxygen saturation (\%) & $78(77-79)$ & $77(77-78)$ & .07 \\
Duration of ventilation (d) & $0.9(0.6-1.3)$ & $0.68(0.3-1)$ & .26 \\
Chest tube duration (d) & $4.2(3.5-4.8)$ & $2.5(2.3-2.7)$ & $<.05$ \\
Chest tube drainage (mL) & $374(302-447)$ & $134(119-150)$ & $<.05$ \\
Chylothorax (n [\%]) & $6(18.1 \%)$ & $2(2.9 \%)$ & $<.05$ \\
ICU stay (d) & $5.5(4.2-6.9)$ & $4.3(3.6-5.1)$ & .1 \\
Length of stay (d) & $10(8-12.7)$ & $7(5.9-8.3)$ & $<.05$ \\
Hospital readmissions (n) & $1.19(0.37-2)$ & $1(0.58-1.42)$ & .64 \\
Age at Fontan (y) & $2.4(2.2-2.7)$ & $2.3(2.1-2.4)$ & .18 \\
\hline
\end{tabular}

Data are presented as mean (95\% confidence intervals) unless otherwise stated. $I C U$, Intensive care unit.

Although our results show a trend toward higher oxygen saturations in patients with a pulsatile Glenn, this difference was not significant. One of the reasons could be that the data on saturations were collected during the immediate postoperative period and often patients with additional pulsatile pulmonary blood flow after the Glenn had a more difficult postoperative course, including longer ventilation times and chest tube drainage. Another reason is that patients with a pulsatile Glenn tended to have smaller pulmonary arteries and vascular beds, thus limiting the benefits of pulsatile flow during the early postoperative period. Indeed, at the preoperative Fontan evaluation these patients were noted to have higher saturations than those without pulsatile pulmonary blood flow.

We noted an increase in chest tube duration and output and a higher incidence of chylothorax in patients who had additional pulmonary flow, presumably due to increased systemic venous pressures. This resulted in a slightly longer hospitalization course after BDG; however, after discharge

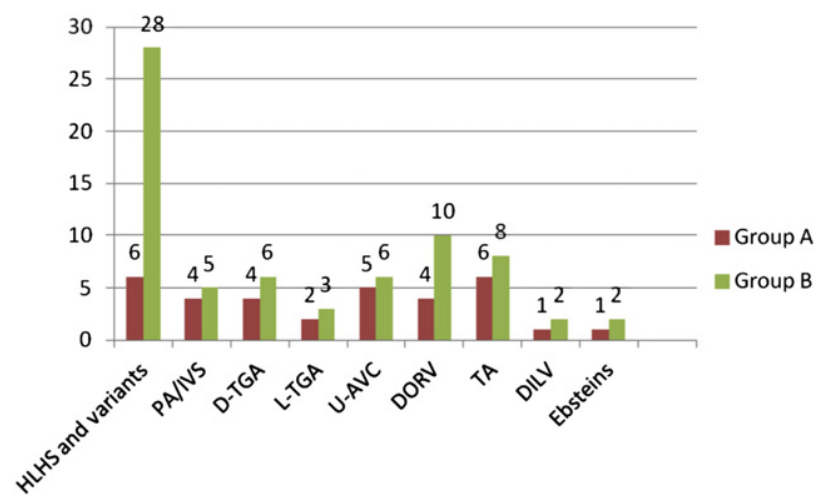

FIGURE 1. Anatomical diagnosis in groups A and B. HLHS, Hypoplastic left heart syndrome; $P A / I V S$, pulmonary atresia and intact ventricular septum; D-TGA, D-transposition of great arteries; $L-T G A$, L-transposition of great arteries; $U-A V C$, unbalanced atrioventricular canal; DORV, double outlet right ventricle; $T A$, tricuspid atresia; $D I L V$, double inlet left ventricle.
TABLE 2. Postoperative echocardiography data

\begin{tabular}{lccc}
\hline \multicolumn{1}{c}{ Parameter } & $\begin{array}{c}\text { Group A } \\
(\mathbf{n}=\mathbf{3 3})\end{array}$ & $\begin{array}{c}\text { Group B } \\
(\mathbf{n}=\mathbf{7 0})\end{array}$ & P value \\
\hline Ventricular function & & & \\
$\quad$ Normal & $23(69.7)$ & $51(72.8)$ & .87 \\
Mildly depressed & $8(24.2)$ & $14(20)$ & \\
Moderately depressed & $2(6)$ & $4(5.7)$ & \\
$\quad$ Severely depressed & $0(0)$ & $1(1.4)$ & \\
AV regurgitation & & & \\
$\quad$ None & $20(60.6)$ & $43(61.4)$ & .92 \\
Mild & $11(33.3)$ & $22(31.4)$ & \\
Moderate & $2(6)$ & $4(5.7)$ & \\
Severe & $0(0)$ & $1(1.4)$ & \\
\hline
\end{tabular}

Data are presented as $\mathrm{n}(\%) . A V$, Atrioventricular valve.

there was no significant increase in hospital readmissions in the pulsatile BDG group. An increase in the incidence of pleural effusion and chylothorax was also noted by Calvaruso and colleagues, ${ }^{6,9,10,15}$ although Caspi and colleagues ${ }^{1}$ did not demonstrate increased rates of persistent pleural effusions with their series of patients.

Exclusion of all other sources of pulmonary blood flow at the time of BDG is aimed at volume unloading the single ventricle, improving its function, and reducing atrioventricular valve regurgitation. ${ }^{16}$ In view of this, an additional source of pulmonary blood flow in a pulsatile BDG may less effectively reduce the volume load on the single ventricle. However, in our series the ventricular and atrioventricular valve function was noted to be comparable in both groups. This is similar to results found by Caspi and colleagues ${ }^{1}$ who demonstrated no adverse effects of additional anterograde pulmonary blood flow at the time of the BDG on ventricular function or atrioventricular valve regurgitation. Previous studies demonstrated higher oxygen saturation. ${ }^{6-8}$ Although a trend was noted in this series, the difference was not statistically significant.

Our series of patients includes 6 patients in whom anterograde pulsatile pulmonary blood flow at the time of the BDG was provided through a banded right ventricle to pulmonary artery conduit (Sano). This was performed because of the diffuse hypoplasia of the pulmonary vascular bed. To the best of our knowledge, this is the first time such an

TABLE 3. Cardiac catheterization data

\begin{tabular}{lrrr}
\hline \multicolumn{1}{c}{ Parameter } & $\begin{array}{c}\text { Group A } \\
(\mathbf{n = 3 3 )}\end{array}$ & $\begin{array}{c}\text { Group B } \\
(\mathbf{n = 7 0 )}\end{array}$ & $\boldsymbol{P}$ value \\
\hline McGoon ratio & & & \\
$\quad$ Pre-Glenn & $1.5(1.46-1.57)$ & $1.59(1.53-1.70)$ & .11 \\
$\quad$ Pre-Fontan & $1.76(1.73-1.79)$ & $1.6(1.53-1.66)$ & $<.05$ \\
Pre-Fontan saturation (\%) & $84(82.4-84.9)$ & $81(80.2-82.6)$ & $<.05$ \\
$\begin{array}{l}\text { Pre-Fontan PA pressure } \\
\quad(\text { mm Hg) }\end{array}$ & $14(12.8-15.2)$ & $10(9.7-11)$ & $<.05$ \\
$\begin{array}{l}\text { Venovenous collateral } \\
\quad \text { pre-Fontan }\end{array}$ & $11 / 33(33.3 \%)$ & $17 / 70(24.3 \%)$ & .28 \\
\hline
\end{tabular}


arrangement of pulsatile Glenn is described. Even in the largest multicenter series of 256 patients described by Calvaruso and colleagues, ${ }^{10}$ anterograde flow through a banded Sano has not been described.

Lower hospital mortality rates have also been reported among patients with antegrade pulmonary blood flow compared with patients in whom bidirectional cavopulmonary anastomosis was the only source of pulmonary blood flow. ${ }^{4,5}$

We did not note any morbidity during the 10-year study period described in our study, especially in patients with small pulmonary arteries. Although there was an increase in the immediate morbidity in terms of increased duration of chest tube output once discharged from the hospital, the readmission rate and rate of recurrence of pleural effusions were comparable for both of our groups. We believe the benefit achieved by increased McGoon ratio at the time of the Fontan outweighs the short-term risks noted in our study. It would be interesting to study pulmonary artery growth after the pulsatile flow has been disrupted following Fontan completion; this is one of the subjects of study of our current project evaluating outcomes after the Fontan surgery.

\section{Limitations}

This study is retrospective and subject to all the inherent bias of a retrospective investigation, including selection of patients for the pulsatile BDG, which was based on subjective and limited objective data as to the size and capacity of the pulmonary vascular bed and potential for bias with reporting of subjective investigation parameters. We recognize the limitations associated with attempting to match our groups for pre- and intraoperative variables, especially with the anatomic and physiologic variability associated with single ventricle patients.

\section{CONCLUSIONS}

Pulsatile BDG is associated with better pulmonary artery growth, which might improve long-term outcomes after Fontan. However, it was associated with a higher postoperative complication rate.
The authors thank Katie Wittle, MSN, RN, for her invaluable help in locating data.

\section{References}

1. Caspi J, Pettitt TW, Ferguson TB Jr, Stopa AR, Sandhu SK. Effects of controlled antegrade pulmonary blood flow on cardiac function after Bidirectional cavopulmonary anastomosis. Ann Thorac Surg. 2003;76:1917-21.

2. Uemura H, Yagihara T, Kawashima Y, Okada K, Kamiya T, Anderson RH. Use of bidirectional Glenn procedure in the presence of forward flow from the ventricles to the pulmonary arteries. Circulation. 1995;92:II228-32.

3. Yoshida M, Yamaguchi M, Yoshimura N, Murakami H, Matsuhisa H, Okita Y. Appropriate additional pulmonary blood flow at the bidirectional Glenn procedure is useful for completion of total cavopulmonary connection. Ann Thorac Surg. 2005;80:976-81.

4. Miyaji K, Shimada M, Sekiguchi A, Ishizawa A, Isoda T. Usefulness of pulsatile bidirectional cavopulmonary shunt in high-risk Fontan patients. Ann Thorac Surg. 1996;61:845-50.

5. Knott-Craig C, Fryar-Dragg T, Overholt E, Razook J, Ward K, Elkins R. Modified hemi-Fontan operation: an alternative definitive palliation for high-risk patients. Ann Thorac Surg. 1995;60:S554-7.

6. Frommelt MA, Frommelt PC, Berger S, Pelech AN, Lewis DA, Tweddell JS, et al. Does an additional source of pulmonary blood flow alter outcome after a bidirectional cavopulmonary shunt? Circulation. 1995;92:II240-4.

7. Berdat PA, Belli E, Lacour-Gayet F, Planché C, Serraf A. Additional pulmonary blood flow has no adverse effect on outcome after bidirectional cavopulmonary anastomosis. Ann Thorac Surg. 2005;79:29-36.

8. Kobayashi J, Matsuda H, Nakano S, Shimazaki Y, Ikawa S, Mitsuno M, et al. Hemodynamic effects of bidirectional cavopulmonary shunt with pulsatile pulmonary flow. Circulation. 1991;84(5 Suppl):III219-25.

9. McElhinney DB, Marianeschi SM, Reddy VM. Additional pulmonary blood flow with the bidirectional Glenn anastomosis: does it make a difference? Ann Thorac Surg. 1998;66:668-72.

10. Calvaruso DF, Rubino A, Ocello S, Salviato N, Guardì D, Petruccelli DF, et al. Bidirectional Glenn and antegrade pulmonary blood flow: temporary or definitive palliation? Ann Thorac Surg. 2008;85:1389-95.

11. Billingsley AM, Laks H, Boyce SW, George B, Santulli T, Williams RG. Definitive repair in patients with pulmonary atresia and intact ventricular septum. J Thorac Cardiovasc Surg. 1989;97:746-54.

12. Fontan F, Fernandez G, Costa F, Naftel DC, Tritto F, Blackstone EH, et al. The size of the pulmonary arteries and the results of the Fontan operation. J Thorac Cardiovasc Surg. 1989;98:711-24.

13. Yin Z, Wang Z, Zhu H, Zhang R, Wang H, Li X. Experimental study of effect of Fontan circuit on pulmonary microcirculation. Asian Cardiovasc Thorac Ann. 2006;14:183-8.

14. Van de Wal HJ, Ouknine R, Tamisier D, Lévy M, Vouhé PR, Leca F. Bi-directional cavopulmonary shunt: is accessory pulsatile flow good or bad? Eur J Cardiothorac Surg. 1999;16:104-10.

15. Mainwaring RD, Lamberti JJ, Uzark K, Spicer RL, Cocalis MW, Moore JW. Effect of accessory pulmonary blood flow on survival after the bidirectional Glenn procedure. Circulation. 1999;100(19 Suppl):II151-6.

16. Berman NB, Kimball TR. Systemic ventricular size and performance before and after bidirectional cavopulmonary anastomosis. J Pediatr. 1993;122(Suppl 6): S63-7. 